\title{
Singular Spectrum Analysis and Neural Network to Forecast Demand in Industry
}

\author{
Rafael Ferreira Lopes, Fabiano Fragoso Costa, Antonio Cezar de Castro Lima \\ Federal University of Bahia \\ Department of Electrical Engineering, Salvador, Brazil \\ rferreir@mtu.edu; acdcl@ufba.br
}

\begin{abstract}
The relationship between energy consumption and supply is a primary factor in the planning and operation of power systems. Brazil is experiencing major problems with the energy crisis into which it was placed. The lack of investment in energy supply is one of the determining factors. During the 1980s, these investments cost $\$ 10$ billion on average every year. In recent years, however, half reduced these investments. This paper proposes a method for demand forecasting based on the Singular Spectrum Analysis (SSA) and neural network. The methods are to be used by large power utility's customers and to be implemented in real-time and prevents peaks from surpassing the contracted power demand with the utility. It can be applied as an auxiliary tool for management of electrical power demand in industrial plants. The effectiveness of the method is endorsed by the high correlation between the forecasted and actual time-series forecasted.
\end{abstract}

Keywords: Singular Spectrum Analysis; Neural Network; Demand Side Management; Forecast

\section{Introduction}

Due problems such as lack of rain shows the reality of the lack of an efficient planning process in the country. The levels of reservoir in the Northeast and Southeast reservoirs have been recorded daily. This shows a need for the state to reinvest in this sector and that conditions are created for the private sector to also invest and make your more efficient consumption.

The problem of consumption efficiency misinformation is still prevalent mainly in small and medium-sized businesses. Many industries don't understand their accounts, for example; how much is spent and how much can be saved.

If the contracted demand is not exceeded, the energy dealers will not have to increase its own demand. This is a benefit to both parties. The energy dealers want to sell as much of the power they generate as possible and the consumer wants to make the most of their money.

The area of Energy Management can be seen as one of the main factors, since a large part of the production process depends on the consumption of electricity by its machinery and equipment to manufacture the final product. The electricity consumption is now one of the parameters used to see whether a country is developed, is developing or is underdeveloped. A methodology that allows for the energy demand with some time in advance would help avoid overtaking in peak times thus producing more efficient consumption with less penalties/drawbacks/disadvantages [2].

The diversification and dynamics of production are variables that directly affect the hiring of electricity demand with a dealership. It is through the supply contract that the technical characteristics and the commercial terms of electricity supply are set to the value demand contracted every month [3].

The electricity bill should be divided into two parts: measured demand and measured consumption. The measured consumption refers to the energy consumed during the month in peak and off-peak hours, being billed separately. However, demand measurement refers to the increase in demand for active power. This is verified by measurement paid-in 15-minute intervals during the billing period in kilowatts (KW).

These measurements are made by electronic meters, necessary for the application of the tariff structure [3]. If the demand value measured, is less than, equal to, or even $10 \%$ higher than the contracted demand of value then one pays the full contracted amount. If the demand value measurement is more than $10 \%$ over the contracted demand value, however, then you pay the contract value together with the fine for registered Overtake value, which is approximately three times the unit price of the kilowatt $(\mathrm{kW})$ of contracted demand. 
When the contracted demand value is greater than the demand value as the false impression one has of the electrical system of the company is well proportioned, it does not pay for exceeding the demand. However, an excess paid by contracting it in fact is not being consumed. This generates an additional cost, which is stipulated in the supply contract and goes unnoticed by the financial sector of the company for not coming broken in the electricity bill.

The development of statistical methods for analysing data obtained in cases where the observations are dependent has shown tremendous growth in recent decades and in particular, the data from time series analysis, which is the case of the series presented here.

When working with time series, the most common goal is predicting future values. The need for accurate predictions of future events and their consequences, whether climatic, economic, epidemiological or of any nature, has led to steady development in the forecasting of techniques in a time series. The classical statistical methods for a time series analysis are well documented. However, many of these methods require specialized knowledge for their correct application. Thus, the proper use of the classic models require checks of their assumptions. This requires effort and experience in exploratory data analysis. The Singular Spectrum Analysis (SSA, Singular Spectrum Analysis of the English), is presented as a relatively simple and powerful alternative that can be applied. However, in some cases a neural network feedforward with 10 or 15 neurons in hidden layer have better performance, therefore, proposal the solution more efficient combination is between these two techniques.

\section{Methodology}

SSA is a nonparametric method used in time series analysis and requires little prior knowledge of the series behaviour. This technique investigates the behaviour of time series through the decomposition and reconstruction of the components that characterize the stages of SSA [1].

The SSA method proves helpful in the series of analyses of the areas of meteorology, geophysics, physics, climatology, economics, health and many other fields of knowledge. This tool can be applied in short or long series, stationary or non-stationary series, noisy or not, or at any time with any number [4] structure [6].

It is noteworthy that the SSA perform of future values predictions, but the technique aims to identify and extract generator standards of the time series.

Fig. 1 shows the time series used in this study, whose data is referred to in the necessary energy demand in a month. Measurements were taken every 15 minutes and only on weekdays.

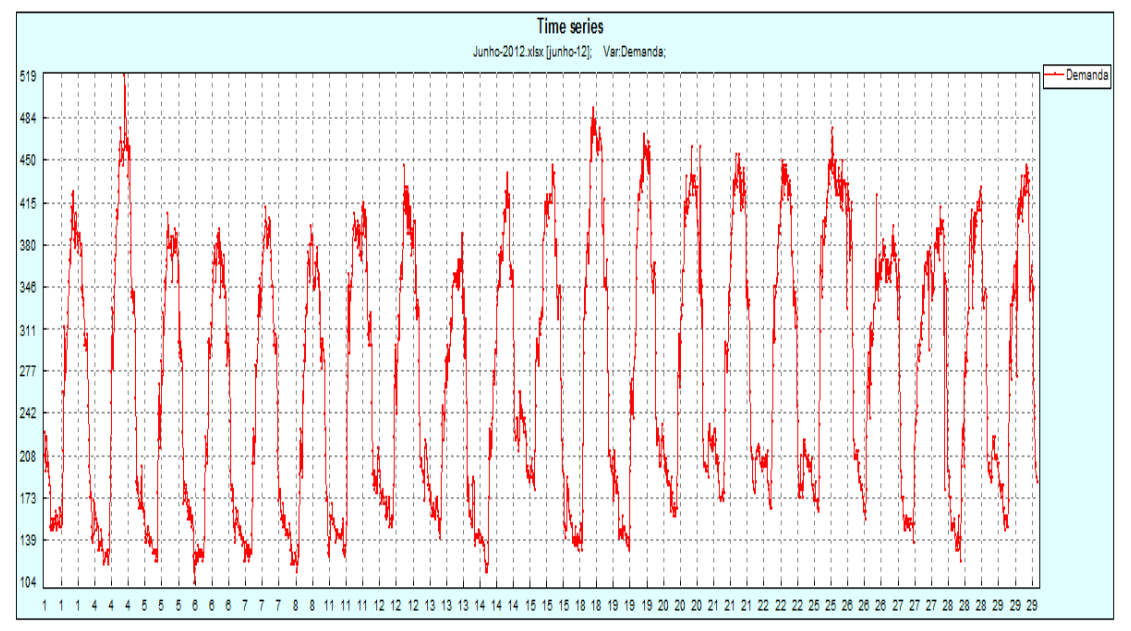

Fig. 1. Time series on energy demand (only on weekdays) in June 2015.

The SSA technique is based on two stages that complement each other: decomposition and reconstruction of the time series. Each stage consists of two steps that form the four steps of the technique: Embedding, Singular Value Decomposition (SVD), Grouping and Diagonal Averaging. The definition of both can be seen in [1]. In addition to the use of SSA technique was used neural network for combination purposes / comparison. 
The backpropagation is a major neural training algorithm. The numerical adjustment synaptic weights, using this algorithm, is by means of a two basic phases optimization process: the forward and backward. In forward phase estimated if the answer provided by the ANN from a set of input patterns, which depends the size of the neural window. In the backward phase error between the response of the ANN and the desired response is used in the process update the synaptic weights. During the training, the input patterns and associated desired responses are presented to the ANN, in order to minimize the mean square error, the training sample, between the output signals of the ANN and the desired signal output. It is possible to perform the prediction of future values of a time series, using an ANN feedforward, with a hidden layer with 'n' neurons and an output layer with a neuron due to approximator character of compact support function used.

Thus, previously set the size ' $L$ ' of the window and the desired ' $h$ ' prediction horizon. In an autoregressive time series with time dependency structure (linear or nonlinear), it has been that the input pattern set is composed of the past values of the time series itself, which is desired design.

The desired output pattern in this case is the value to be predicted.



Fig. 2. Artificial Neural Network with $\mathrm{L}=4$.

Fig. 2 is the illustration of the dynamic prediction, one step ahead of a time series with self-reliance structure with a lag equal to 4 (i.e., L is equal to 4) through a feedforward ANN. Note that the input pattern vector comprises the last 4 temporal signals before the output pattern to be expected. To predict the subsequent signal, the ANN window it is shifted one-step ahead.

\section{Results and Discussion}

Through applying the step of decomposing the series electrical energy demand data, we found the following results: In Fig. 3, one can observe that the unique value of the first eigenvector is considerably larger than the other singular value eigenvectors. Thus, it can be said that the eigenvector 1 is well separated from the other eigenvectors and is the main trend of the series. The eigenvectors 2 and 3 have very close singular values, forming a step on the graph and therefore representing a harmonic component. Based on the graph it can also be said that the eigenvectors, possibly 4, 5, 6 and 7 forming the lower step, also represent a harmonic component. As for the others, they still cannot claim to be noise.

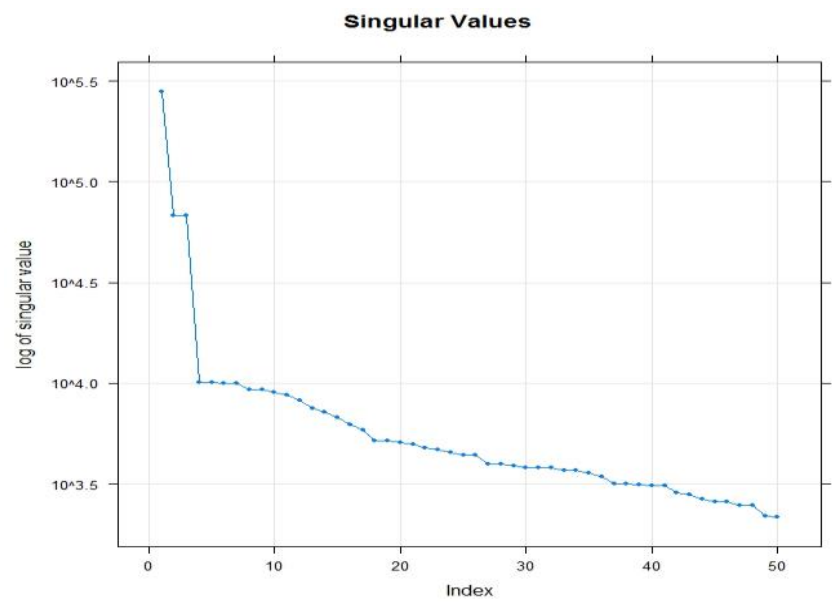

Fig. 3. Logarithm of the first 50 eigenvectors of the SVD. 
In the graph of the average covariance between the eigenvectors, shown in Fig. 4, there is the periodic behaviour of the covariance functions of the original series. The distance, in the axis X, among the largest covariance values, as well as among lower, is equal to 96 and is the main frequency of the series. The graph of covariance confirms the initial result of the frequency shown in the visual analysis of the original time series.

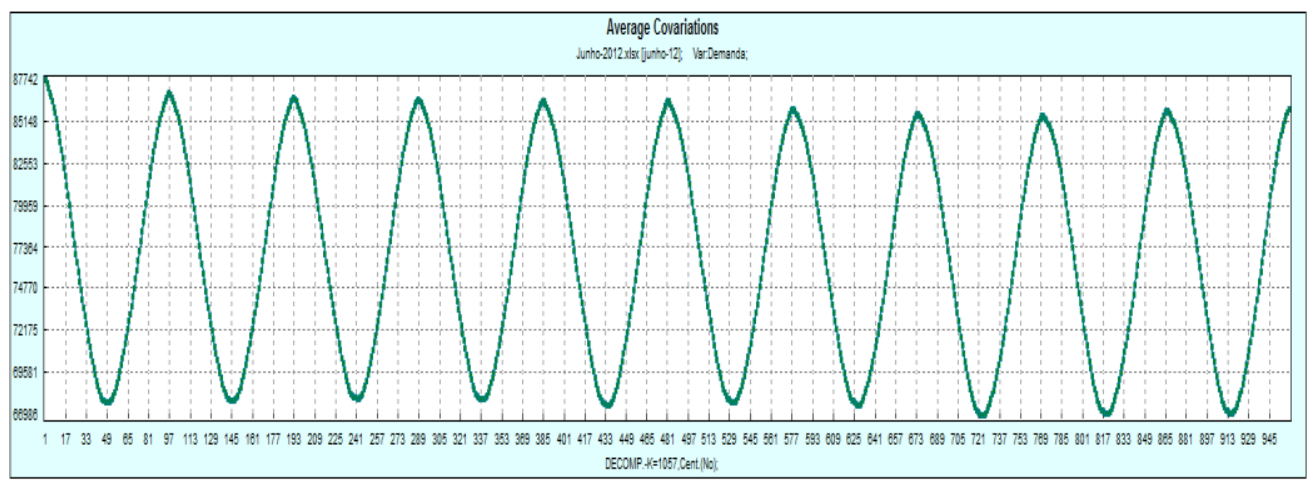

Fig. 4. Covariance average of eigenvectors.

Additional information regarding the frequency is given in the periodogram analysis, where we observe the extracted seasonal components of the time series. However, only one harmonic frequency component, equal to the periodogram, is distinguishable from 96 shown in Fig. 5 .

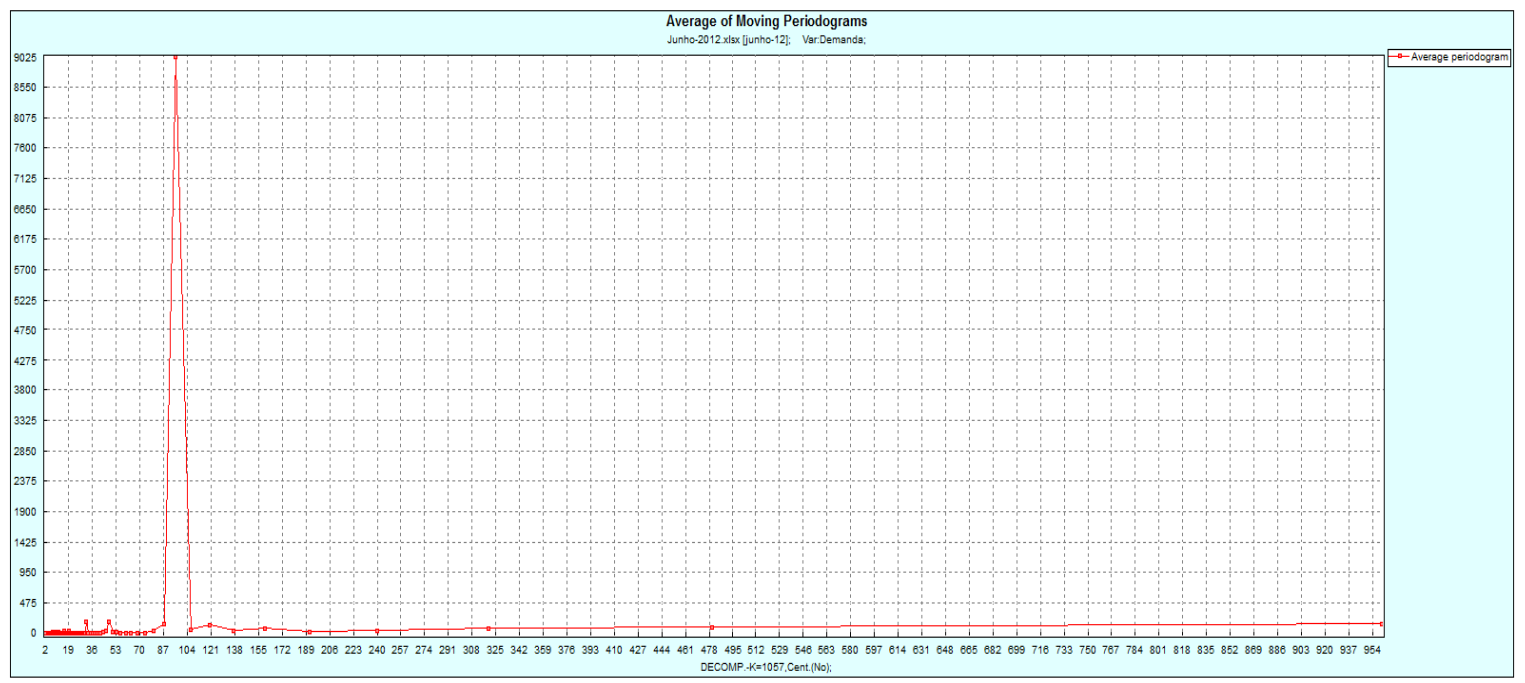

Fig. 5. Average periodogram of the time series.

In Fig. 6 the first nine natural eigenvectors of the matrix path SVD can be observed. Through this observation, it can be seen that the component SSA 1 (which is associated with the unique eigenvector 1, SVD) is equal to 87.819\%. Also based on the plot of eigenvalues and Figure 3 it can be concluded that the SSA component 1 was confirmed as the main trend and none of the other components, such as harmonics, were considered according to noise [4].

After the step of decomposition the reconstruction stage was started. Depending on the mix between the harmonic components, trend and noise, it was often difficult to decide which eigenvectors should be included in the reconstruction of the series. Therefore, the evaluation of the weighted correlation matrix was necessary. Looking at Fig. 7 , it can be noted that the pairs of eigenvectors which derived from the same trend component are highly correlated, such as the eigenvalues 2 and $3(\rho=1.000)$, and the harmonic component from the period is equal to 96 , which are eigenvectors 4 and 5 ( $\rho=$ $0.964)$ and peer 6 and $7(\rho=0.970)$. The first eigenvector, which is the main trend in the series, does not correlate with any 
other eigenvector which also shows that the signal-to-noise separation was conducted efficiently. The eigenvectors 10 and 11 are correlated, although this correlation is not as high $(\rho=0.919)$ and the correlation between the other eigenvector harmonics.

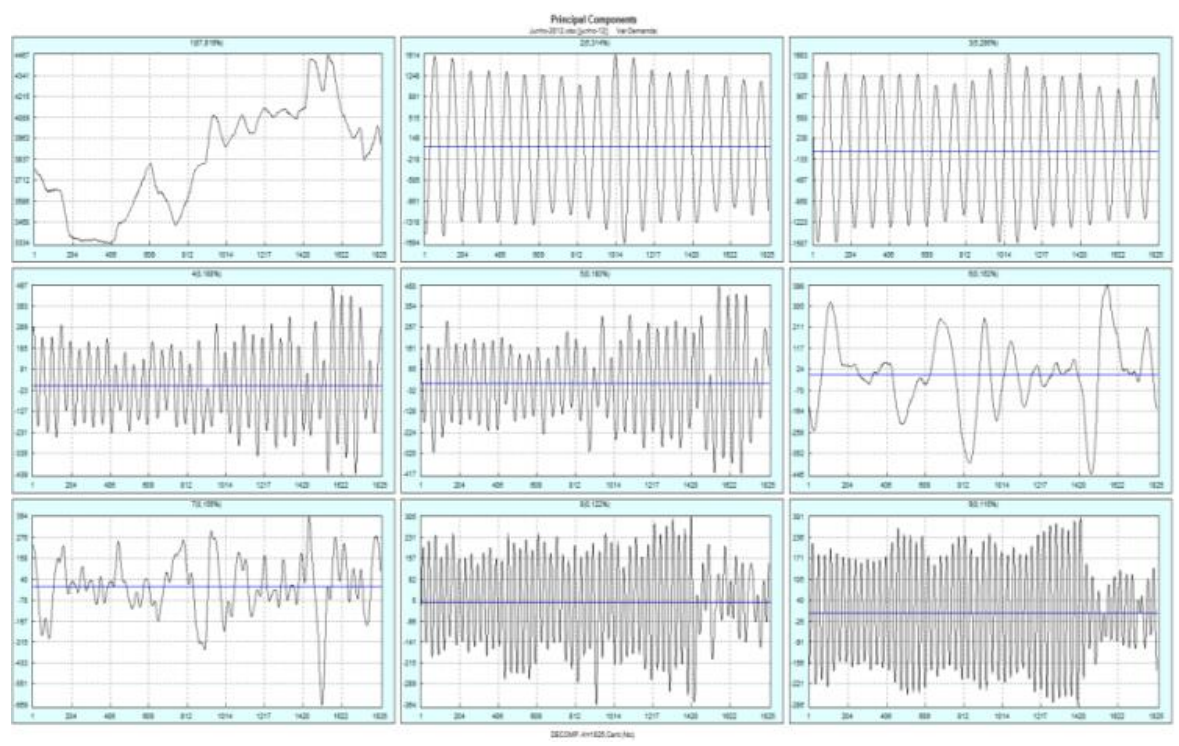

Fig. 6. First singular eigenvectors in the matrix path SVD

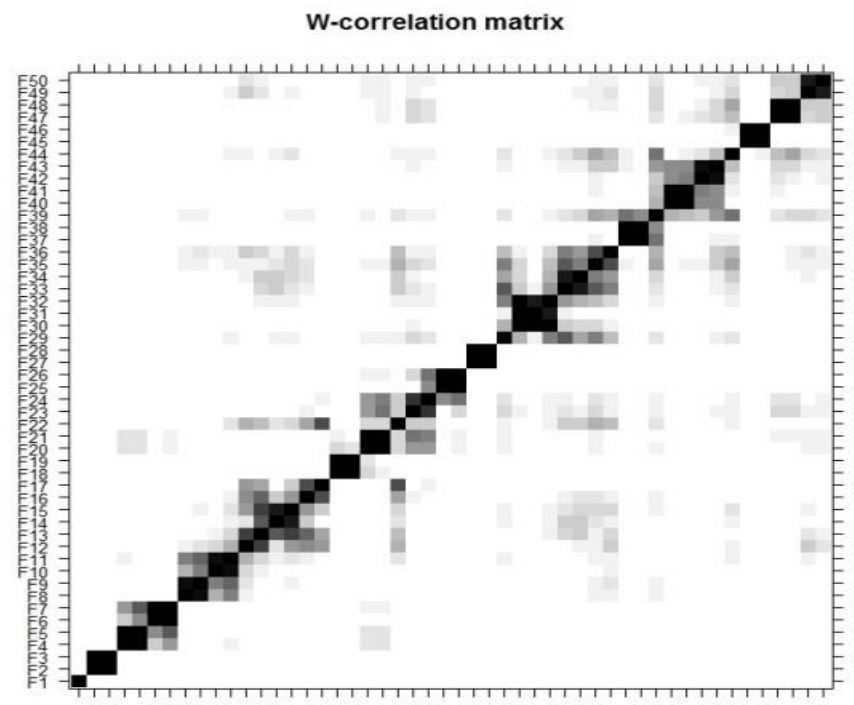

Fig. 7. Correlation Weighted Matrix.

As the singular values of the eigenvectors, 7 and 8 are not very close, we chose to include these eigenvectors in the reconstruction of the series trend. Eigenvector 18 and the subsequent eigenvectors have different correlations with other eigenvectors, indicating similarities between the eigenvectors mixture. These eigenvectors were interpreted as originating from noise in the time series.

Grouping the trend and seasonality of the time series (1-17), it is possible to reconstruct the signal of the series, as can be seen in Fig. 8.

The highest residue found in the reconstruction stage was $118 \mathrm{~kW}$. While much of the error can be attributed to noise present in the original series, it can be said that the error in reconstruction can also be due to extreme events and 
unpredictability .The absolute average error (MPE) and the mean relative error (RMS) were equal to $17.6 \mathrm{~kW}$ and $0.9 \%$, respectively, and furthermore, the original series and the reconstructed series are significantly correlated $(\rho=0.9124 ; \mathrm{t}=$ $38.8962, \mathrm{df}=550, \mathrm{p}$-value $<2.1 \times 10-16)$. The absolute errors in the reconstruction step can be further seen in Fig. 8 .

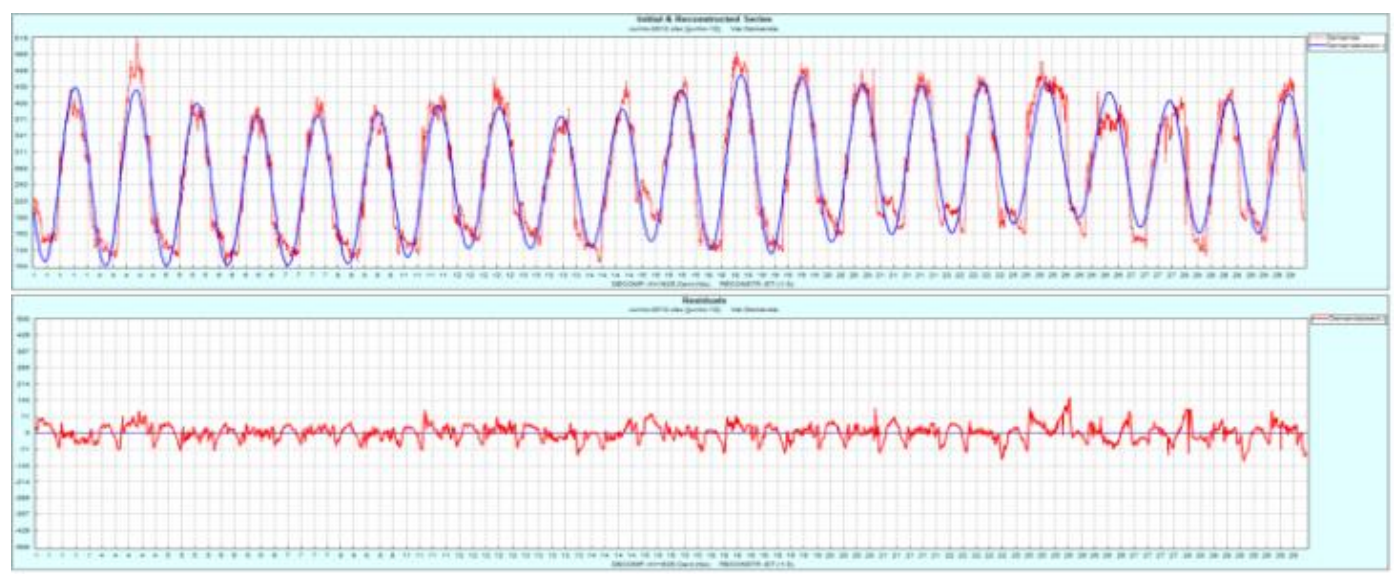

Fig. 8. Original Series x Rebuilt Series and Waste.

After the series be reconstructed, prediction tests were performed using recursive algorithm of the SSA technique and neural networks 10 and 15 neurons in the hidden layer.

The results were compared using metrics such as Mean Absolute Percentage Error (MAPE), Mean Squared Error (MSE) and Total Percentage Error (TPE) in Fig. 9.

\begin{tabular}{|c|c|c|c|c|c|c|c|c|c|}
\hline \multirow{2}{*}{$\mathrm{h}$} & \multicolumn{3}{|c|}{$\begin{array}{c}\text { Neural Network FF } \\
10 \text { Neurons }\end{array}$} & \multicolumn{3}{c|}{$\begin{array}{c}\text { Neural Network FF } \\
15 \text { Neurons }\end{array}$} & \multicolumn{2}{c|}{ Recursive Algorithm of SSA } \\
\cline { 2 - 10 } & MAPE & MSE & TPE & MAPE & MSE & TPE & MAPE & MSE & TPE \\
\hline 1 & $4.36 \%$ & 73,51 & $4,36 \%$ & $4,53 \%$ & 79,19 & $-4,53 \%$ & $39,89 \%$ & 6136,84 & $-39,89 \%$ \\
\hline 4 & $8,09 \%$ & 251,14 & $-8,05 \%$ & $9,73 \%$ & 375,52 & $-9,67 \%$ & $44,49 \%$ & 7161,25 & $-44,44 \%$ \\
\hline 12 & $6,86 \%$ & 233,42 & $0,66 \%$ & $6,83 \%$ & 250,41 & $-6,04 \%$ & $26,18 \%$ & 3566,89 & $-25,26 \%$ \\
\hline 24 & $5,64 \%$ & 158,82 & $0,78 \%$ & $14,93 \%$ & 1214,78 & $-14,06 \%$ & $20,07 \%$ & 2178,75 & $-6,16 \%$ \\
\hline 48 & $14,77 \%$ & 3178,57 & $8,35 \%$ & $26,58 \%$ & 3525,18 & $-14,46 \%$ & $16,46 \%$ & 1554,00 & $-3,49 \%$ \\
\hline 72 & $27,64 \%$ & 17446,0 & $30,41 \%$ & $29,46 \%$ & 9457,91 & $19,94 \%$ & $19,31 \%$ & 4501,27 & $-13,76 \%$ \\
\hline 96 & $31,50 \%$ & 21690,4 & $35,54 \%$ & $29,71 \%$ & 11176,62 & $15,01 \%$ & $18,75 \%$ & 4273,75 & $-14,31 \%$ \\
\hline
\end{tabular}

Fig. 9. Results of the MAPE, MSE and TPE.

\section{Conclusion}

This method allowed us to determine the trend characteristic of the series, extract seasonal components, and separate the components that represent the signal (information) of the noise. In the case of the data analysed during the month of June in this study, the method was effective in extracting the trend component and seasonal components of the entire series. The series had at least a seasonal component for the period of 96 minutes. The analysis of eigenvectors, periodogram and covariance medium confirmed the seasonality due to the daily cycle characteristic of the industry. 
Taking into account the magnitude of the errors and the correlation coefficient, it can be concluded that the reconstructed series with the first 10 and the extracted eigenvectors represented the behaviour of the original series. In addition to revealing seasonal variations, another relevant fact regarding the robustness of the method is its ability to reveal trends whose behaviour is nonlinear. The results show that spectral analysis is a useful technique when you want to extract information about the behaviour of time series in order to reveal their trends and seasonal effects.

However, analysing the errors is possible to say that for short-term prediction performed by a neural network is more efficient, however, from a more distant horizon using recursive algorithm of the SSA becomes more efficient. Using the combination this method it was especially possible to predict the demand that exceeded the contracted peaks in almost all cases, thus reducing costs and maximizing efficiency of use.

\section{Acknowledgements}

In the Support Foundation of Bahia State Research (FAPESB) for financial support.

\section{References}

[1] R. F. Lopes, F. F. Costa, A.C. de C. Lima, "Industry Energy Demand Forecast in Real Time via Singular Spectrum Analysis," Przeglad Elektrotechniczny, ed. December 2015, pp. 21.

[2] Mirosław Parol and Paweł Piotrowski, "Long-term forecasting method of annual electrical energy demand in electric distribution companies," Przeglad Elektrotechniczny, ed. Oct. 2010, pp. 182.

[3] ANEEL - Agência Nacional de Energia Elétrica (Brasil). (2015, September 11). Resolução n ${ }^{\circ} 456$, de 29 de novembro de 2000.Art. $2^{\circ}$, II. [Online]. Available: http://www.aneel.gov.br/cedoc/res2000456.pdf.

[4] N. Golyandina, V. Nekrutkin, and A. Zhigljavsky, Analysis of Time Series Structure: SSA and Related Techniques. Chapman Hall/CRC, New York, 2001.

[5] N. Golyandina, E. Osipov, "The 'Caterpillar'-SSA method for analysis of time series with missing values," Journal of Statistical Planning and Inference, no.137, pp. 2642-2653, 2007.

[6] H. Hassani, "Singular Spectrum Analysis: Methodology and Comparison," Journal of Data Science, no.5, pp. 239$257,2007$.

[7] H. Hassani, S. Heravic, A. Zhigljavsky, "Forecasting European Industrial Production with SSA," International Journal of Forecasting, no. 25, pp. 103-118, 2009.

[8] R. M. Esquivel, "Análise Espectral Singular: Modelagem de séries temporais através de estudos comparativos usando diferentes estratégias de previsão," Dissertação de Mestrado em Modelagem Computacional e Tecnologia Industrial, CIMATEC/SENAI-BA, Salvador, pp. 173, 2012. 